\title{
A brief review on Drug-induced hepatotoxicity: Use of hepatoprotective agents
}

\author{
Ervilla Dass
}

Associate Professor, Dept. of Pharmacology, Smt. B. K. Shah Medical Institute \& Research Centre, Sumandeep Vidyapeeth An Institution Deemed to be University, Vadodara, India

*Corresponding Author: Ervilla Dass

Email: ervilladass@gmail.com

\begin{abstract}
Adverse Drug Reactions (ADRs) are common and constitute major health problem. During the last decade liver toxicity has been one of the most frequent reasons for pharmacovigilance safety reports and the withdrawal from the market of an approved medicinal product. Liver is a major organ involved in the systemic detoxification and deposition of endogenous and exogenous substances. Liver dysfunction challenges not only health care professionals but also the pharmaceutical industry and drug regulatory agencies. Drug-Induced Liver Injury (DILI) also referred as drug-induced hepatotoxicity, forms a major clinical problem, which has become the leading cause of acute liver failure and transplantation in Western countries. Drug-induced hepatotoxicity is one of the major causes of acute and chronic liver disease. It is also the most common adverse event that halts the development of a new drug or leads to the withdrawal of approved drugs from the market. Therefore, there is a need for guidance on how to act when non-clinical and/or clinical signals of hepatotoxicity are detected in order to improve human safety. The present review study was done by extensive literature search using the keywords such as, Adverse Drug Reactions, Drug-Induced Hepatotoxicity, Hepatoprotectives, Hepatotoxicity, Liver Injury, Methionine and N-Acetyl -L-Cysteine. The current therapy for paracetamol-induced hepatic toxicity is N-acetyl L-cysteine (NAC), which is a cysteine prodrug and GSH precursor and is often given as a treatment for paracetamol overdose. N-acetyl L-cysteine must be administered within eight hours of paracetamol overdose for maximum efficacy. DL-methionine also has been proved to have hepatoprotective effect in paracetamol-induced hepatotoxicity.
\end{abstract}

Keywords: Adverse Drug Reactions, Drug-Induced Hepatotoxicity, Hepatoprotectives, Hepatotoxicity, Liver Injury, Methionine, N-Acetyl -L-Cysteine.

\section{Introduction}

Adverse Drug Reactions (ADRs) are common and constitute major health problem. Liver being the major organ involved in the systemic detoxification and deposition of endogenous and exogenous substances; the disease of liver are considered to be serious health disorders, continues to be one of the major cause of morbidity and mortality rate which encompasses a clinical histological spectrum. The parent drug administered is often responsible for producing the desired therapeutic effect; but the adverse events or the toxic effects produced by the drugs are not just related to the parent compound where the metabolites of the drug produced by the enzymes, light or by the Reactive Oxygen Species (ROS), contribute to the damage caused, as seen in the toxic reactive intermediate - a metabolite of acetaminophen which being highly reactive binds to nucleophiles such as glutathione (GSH) and in cases when the glutathione is depleted, binds to the cellular macromolecules, thus causing the death of liver cells. ${ }^{\mathbf{1 - 3}}$ Looking to these facts, and importance of drug-induced liver injury the present review study was done by extensive literature search using the keywords such as, Adverse Drug Reactions, Drug-Induced Hepatotoxicity, Hepatoprotectives, Hepatotoxicity, Liver Injury, Methionine and N-Acetyl - LCysteine.

\section{Types of hepatotoxic reactions caused by the drugs}

A variety of hepatic abnormalities caused by the administered drug can comprise the whole range of liver lesions and the adverse effects caused may include Type A (augmented) or Type B (Bizzare) reactions. The augmented reactions can include either/or interference with bilirubin metabolism and its excretion (Rifampicin impairs the hepatic uptake and excretion of bilirubin); centrilobular necrosis (Paracetamol in overdose); hepatocellular necrosis (Salicylates); \& fatty changes in liver (Tetracyclines). While, the Type B (Bizzare) reactions includes; acute hepatocellular necrosis (Carbamazepine, Sodium valproate, Isoniazid, Ibuprofen) \& cholestatic hepatitis (Phenothiazines). . 2, 4

The reactions caused by the drug may be pharmacological (excessive depression of Central Nervous System that is caused by Barbiturates); pathological (hepatic injury produced by Paracetamol); or genotoxic, (neoplasm produced by Nitrogen mustard). Of these reactions, the pharmacological toxicity or the adverse event can be overcome by reducing the concentration of drug or the chemical in the tissues either by metabolism or excretion from the body. $1,2,4$

Therefore, due to the central role of the liver in the metabolism of drugs liver injury has been documented as one of the most likely complication of majority of the prescribed drugs. Adverse reactions pertaining to liver can be of mainly two types, predictable or dose dependent and unpredictable or idiosyncratic. It has been observed that, even though many drugs associated with a significant risk of hepatotoxicity have been replaced by apparently safer drugs, drug reactions related to liver are still being increasingly reported and documented through numerous studies done by researchers 5 . Hepatotoxicity is reported to be one amongst the more frequently encountered adverse reaction in postmarketing studies or even after, once the drug has already 
been launched; due to the fact that premarketing recruits a relatively low number of patients, frequently insufficient to fully judge the true incidence of hepatotoxicity. 1,6

Therefore, looking to the various studies $\mathbf{3 , 6 , 7 , 8 , 9 , 1 0 , 1 1}^{3}$ it is understood that hepatotoxicity which is also known as Drug-Induced Liver Injury (DILI), is of major concern owing to the ever increasing number of new drug molecules introduced into the pharmaceutical market for treatment of various diseases. Also, the increasing popularity of herbals, which contributes themselves to self-medication by the patients, but is not rigorously regulated, also adds to the cause of DILI and it is found that DILI due to prescription, over-the-counter, and herbal products is a major cause of liver disease in the United States (US) and around the world $^{12}$. Animal models undoubtedly screen out most potent toxins; however liver injury at extremely high doses in animal models does not necessarily predict problems in humans. There is no animal testing in old age, malnutrition, comorbidity or polypharmacy, which are all part of ageing and the frailty syndrome in humans ${ }^{\mathbf{1 0}}$. Recently, in order to improve the outcomes, attempts have been made by the National Institutes of Health (NIH) and the U.S. Food and Drug Administration (FDA) and they have been directed toward a better understanding of these occurrences of drug induced liver injury. ${ }^{6,13}$

\section{Impact of hepatotoxicity on new drug development and importance of post marketing surveillance}

During the last decade liver toxicity has been one of the most frequent cause for pharmacovigilance safety reports and the withdrawal from the market of an approved medicinal product. In the process of a new drug development, due to the limitations on toxicity studies and clinical trials, which are not always adequate to decide which drugs are hepatotoxic, before introduced into the market for the patient's use. Hence though rare, but Druginduced hepatotoxicity will continue with new drug molecules coming on to the market and with established hepatotoxins. Examples of drugs have been withdrawn due to hepatotoxicity are troglitazone, bromfenac, ticrynafen, benoxaprofen, trovafloxacin, ebrotidine, nimesulide, nefazodone, ximelagatran and pemoline4. Therefore, it becomes vital to detect the uncommon yet significant adverse drug reactions through post-marketing surveillance.

Equally, it becomes a must for the physician to assess whether the signs and symptoms that are caused in patients are due to the administered pharmacotherapeutic agents, to identify the adverse effects, so that they may be prevented and thus improve the prognosis, reduce the morbidity, mortality and economic burden in patient healthcare management. ${ }^{4}$

\section{Drug-induced Hepatotoxicity: A Glance}

The Drug-Induced Liver Injury (DILI) was found to have been associated with several risk factors that included, race, alcohol ingestion, pre-existing liver disease, genetic factors, drug formulations and several host factors, such as, gender, age, nutritional status, body mass index, metabolic disorders, renal failure, hepatitis $\mathrm{C}$ and Acquired Immunodeficiency Syndrome (AIDS). ${ }^{1}$ In the past five years, two drugs have been withdrawn from the market by the Food and Drug Administration (FDA) for causing severe liver injury, a potential danger that had not been fully recognized in the course of the preapproval clinical trials. Reports of adverse drug reactions of any type evokes fear and disbelief in the public about the actions of the pharmaceutical industry and the FDA. ${ }^{6}$ In the present situation, Drug-induced hepatic injury is reported to be one of the most frequent reason cited for the withdrawal from the market of an approved drug, and it also accounts for more than $50 \%$ of the cases of acute liver failure in the United States ${ }^{4}$. More than $75 \%$ of cases of idiosyncratic drug reactions has been reported which result in liver transplantation or death ${ }^{4}$. Critical assessment of these cases has led to the observation that the nonclinical data available at the time of marketing application sometimes contained signals that (in retrospect) could have predicted hepatotoxicity ${ }^{4}$.

Drug-induced hepatotoxic reactions are of multiple types and origin and the time to onset varies from being very short to exhibiting a long latency. ${ }^{14}$ Clinically, the most relevant reactions include liver necrosis, hepatitis, cholestasis, vascular changes and steatosis. It is worth emphasizing that species differences in the metabolism of drug \& target drug must be taken into account in the interpretation of findings and in assessing the relevance of such findings to humans. For example, steatosis has significant implications to clinicians i.e., Non-Alcoholic Steato-Hepatitis (NASH), yet it is generally a less important finding non-clinically, particularly if observed in rodents. ${ }^{1,6}$

\section{Drugs causing hepatotoxicity: Incidence and Prevalence}

Most recent examples of hepatotoxic adverse drug reactions that have contributed to the decline of many promising therapies includes bromfenac and troglitazone. The spectrum of Nonsteroidal Anti-Inflammatory Drug (NSAID)-related liver toxicity continues to expand ${ }^{1,15}$ with reports in children, interactive toxicity in persons with hepatitis $\mathrm{C}$, and recognition of the toxicity of both the preferential and selective cyclooxygenase-2 inhibitors. ${ }^{4,16}$ Cases of liver injury associated with the Angiotensin Receptor and Converting Enzyme Inhibitors are increasingly reported. ${ }^{4}$ Of the antidiabetic agents, Acarbose, Gliclazide, Metformin, and Human Insulin have been implicated in causing liver injury. ${ }^{4}$ To date, the newer Thiazolidinediones do not appear to share the hepatotoxic potential of Troglitazone, although a few reports of acute hepatitis have accrued. Although liver injury has been associated with the "statins," the frequency of such toxicity is lower than that of the background population and the value of biochemical monitoring remains unproved ${ }^{4}$. Newer concepts in anticonvulsant hepatotoxicity have been the recognition of the reactive metabolite syndrome, delineation of the risk factors for Valproic Acid toxicity, the potential role of Carnitine in preventing Valproic Acid hepatotoxicity, and the toxicity of second-line antiepileptic 
drugs. Liver injury associated with newer psychotropic agents, particularly the Selective Serotonin Reuptake Inhibitors (SSRIs). ${ }^{4,16}$ There are evidence for reactive metabolite formation from hepatotoxic drugs, such as Acetaminophen, Tamoxifen, Diclofenac ${ }^{1,5}$ \& Troglitazone, and report of how this leads to liver injury. Moreover, oxidative stress and diminished glutathione may also play critical roles in the pathogenesis of liver disease. ${ }^{16}$

The incidence of serious adverse drug reactions also increases with increasing age, even after controlling for increased medication use [Moore et al. 2007] $]^{6,17,18}$. Most adverse drug reactions (ADRs) in older people, including drug induced liver injury (DILI), are dose-related [Routledge et al. 2004]. ${ }^{17}$ Presently, hepatotoxicity or cardiac toxicity is the leading cause of withdrawal from the market or termination in drug development in phase IIII. $6,7,18$

The leading cause of drug non-approval and drug withdrawal by the Food and Drug Administration (FDA) in the US is drug-induced hepatotoxicity (Ostapowicz et al., 2002; Pandit et al., 2012). More than a thousand medicines and chemicals have been reported to cause liver injury (Larrey, 2000; Biour et al., 2004; Upadhyay et al., 2010b; Porceddu et al., 2012). Drug-induced liver injury may account for approximately $10 \%$ of all cases of acute hepatitis, 5\% of all hospital admissions, and 50\% of all acute liver failures (Pandit et al., 2012). It is remarkable that more than $75 \%$ of cases of idiosyncratic drug reactions result in liver transplantation or death (Ostapowicz et al., 2002; Pandit et al., 2012). Drug-induced liver injury is a relatively common cause of acute liver disease and carries a mortality of around 10\% (Lewis and Zimmerman, 1989; Shapiro and Lewis, 2007; Chalasani et al., 2008; Bell and Chalasani, 2009; Holt and Ju, 2010; Upadhyay et al., 2010b; Pandit et al., 2012; Bjornsson et al., 2013). ${ }^{3,6,7,13,20}$

\section{Experimental Models of Hepatotoxicity}

Some drugs and chemicals with which is associated hepatotoxicity are employed as inducers of hepatotoxicity in testing for various hepatoprotective agents. Although individual analgesics rarely induce liver damage due to their widespread use, NSAIDs have emerged as a major group of drugs exhibiting hepatotoxicity. ${ }^{11}$ Both dose-dependent and idiosyncratic reactions have been documented (Manov et al., 2006). ${ }^{7}$

\section{Treatment of Hepatotoxicity}

Several enzymes that trigger important chemical reactions in the body are produced in the liver and are normally found within the cells of the liver. However, if the liver is damaged or injured, the liver enzymes spill into the blood, causing elevated liver enzyme levels. The liver enzymes like transaminases, alkaline phosphatase, $\gamma$-glutamyl transpeptidase, sorbitol dehydrogenase, glutamate dehydrogenase and lactate dehydrogenase in the blood can be measured to know the normal functioning of liver. These enzymes help in detecting injury to hepatocytes. In case of patients showing hepatotoxicity with elevated liver enzymes due to certain hepatotoxicant, the enzymes levels usually return to normal within weeks or months after stopping the exposure to the hepatotoxicant which is suspected of causing the problem.

Hepatic failure can cause the destruction of homeostasis which can lead to a human life crisis. Therefore, novel alternative strategies to overcome these limitations are urgently needed. Over the last 20-30 years, different methods have been developed to improve or replace, at least temporarily, essential hepatic metabolic functions. ${ }^{20}$ These have included extracorporeal bioartificial liver devices. ${ }^{21-23}$ and cell therapy. ${ }^{24-26}$ Liver transplant is the only definitive cure, but there is a huge discrepancy between the need for transplantation and the availability of donor organs. As a result, a substantial number of patients die while on the waiting list. Recently, this has promoted much research into living liver tissue support to perform various hepatic functions, but, the development of bioartificial liver support is remarkably delayed compared to the development of artificial kidney and artificial hear. $\mathrm{t}^{27}$

The treatment of hepatotoxicity is dependent upon the causative agent, the degree of liver dysfunction and the age and general health of the individual. Treatment for druginduced hepatotoxicity generally consists of withdrawing the drug and providing supportive therapy. Many hepatoprotective herbal preparations ${ }^{28,29}$ have been recommended in alternative systems of medicine for the treatment of hepatic disorders. There are over 100,000 defined traditional Chinese medicine therapies and $80 \%$ of these are herbal Combinations. ${ }^{28,29}$ Traditional medicine has a long history. It is the sum total of the knowledge, skills and practices based on the theories, beliefs and experiences indigenous to different cultures, whether explicable or not, used in the maintenance of health, as well as in the prevention, diagnosis, improvement or treatment of physical and mental illnesses. The terms complementary/alternative/non-conventional medicine are used interchangeably with traditional medicine in some countries. $^{30}$

The main limit of applicability of Complementary and Alternative Medicine (CAM) therapies comes from the lacking of randomized, placebo-controlled clinical trials giving a real proof of efficacy of those products, so that anecdotal success and personal experience are frequently the driving force for acceptance of CAM in the population. ${ }^{31}$

Moreover, physicians used to focus attention on potential toxicities, even though identification of toxicity from herbal preparations is often difficult, because patients generally self-medicate with these and may withhold this information. Toxic hepatitis is the most common adverse reaction resulting from the use of CAM, often associated with the concomitant consumption of hepatotoxic ingredients such as acetaminophen and nonsteroidal antiinflammatory agents or with hepatotoxicity of herbal ingredients themselves. In contrast to pharmaceuticals, CAM are not evaluated formally for safety and efficacy; variations in methods of harvesting, preparing, and extracting the herb, which can result in dramatically 
different levels of certain alkaloids. The biologically active substances have been structurally defined and standardized for only a few of the herb: in most countries, their use is neither regulated nor controlled ${ }^{31}$. The diagnosis of druginduced liver injury is done by exclusion, and in all cases of liver damage where there is no determinable underlying cause, drug toxicity should be considered. The diagnosis is also based on the temporal association between the onset of damage and the timing of drug exposure, the response to drug withdrawal, and the known pattern of drug reactions ${ }^{32}$. Hepatoprotective agents such as Silymarin, Methionine, Picroliv, Pentoxifylline, Beta-Carotene either of them can be employed as a hepatoprotective agent ${ }^{33}$. Sulfoadenosylmethionine and Silymarin are also promising drugs utilized mainly in cholestasis but the benefits can be expanded if more controlled trials are performed.

Based on the mechanistic insight gained from early preclinical studies (Jollow, 1973; Mitchell et al., 1973a; Mitchell et al., 1973b) N-acetylcysteine was introduced in the 1970s and still is the only clinical antidote against paracetamol-induced liver injury (Polson and Lee, 2005a). Based on a recent review study, NAC has already been established as a safe and effective treatment for paracetamol-induced liver injury. Based on the most recent study which suggests that it is important to review the evidence of NAC safety and efficacy to determine if NAC may be considered as a treatment option in non-paracetamol DILI (Chughlay et al, 2015), ${ }^{3,34-36}$

Prompt use of $\mathrm{N}$-acetylcysteine after acetaminophen overdose and intravenous carnitine for valproate-induced hepatotoxicity has been reported for the treatment of acute liver injury. Nutrient supplements like taurine, methionine, S-adenosylmethionine, arginine, polyenylphosphatidylcholine, $\alpha$-lipoic acid, vitamin $B$, antioxidant vitamins $[\mathrm{A}, \mathrm{C}, \mathrm{E}]$ and methylsulfonylmethane that support phase I and phase II activities also serve as hepatoprotective agents, including N-Acetylcysteine which is the antidote for paracetamol-induced hepatotoxicity. There are many herbs and herbal drugs which are extensively investigated natural products for hepatoprotection such as silymarin, resveratrol, curcumin and gingko ${ }^{19}$. Preclinical tests of various therapeutic agents should be done scientifically. Public should be properly educated about the probability of various hepatotoxicants. Due emphasis should be given on the possibility of drug interactions and sharing knowledge of newly reported hepatotoxins. ${ }^{37}$ All the therapeutic agents should be properly labelled and the dose standardized. Regular monitoring of liver function tests should be done.

Hence, looking to the impact of drug-induced hepatotoxicity, it becomes imperative to know about its various mechanisms, also, oxidative stress and diminished glutathione play critical roles in the pathogenesis of liver disease. Hence, it the need of an hour to do further research in the field of hepatoprotection. As it is now understood about the synthesis of glutathione, the most abundant mammalian antioxidant, is regulated at the substrate level by cysteine, which is synthesized from homocysteine via the trans-sulfuration pathway ${ }^{3}$ various research studies can be done in future using different experimental models of hepatotoxicity. ${ }^{35,36}$ It can be thus concluded that though various research studies have contributed greatly to our understanding of liver injury, they are lacking a more quantitative approach that would provide the data necessary for developing appropriate health policy.

\section{Acknowledgments}

Author is grateful to Smt. Bhikhiben Kanjibhai Shah Medical Institute \& Research Centre, Sumandeep Vidyapeeth an Institution deemed to be University, Piparia, Vadodara, for permitting to conduct the review study.

\author{
Abbreviations \\ Adverse Drug Reactions (ADRs) \\ Drug-Induced Liver Injury (DILI) \\ $\mathrm{N}$-acetyl L-cysteine (NAC) \\ Reactive Oxygen Species (ROS) \\ Glutathione (GSH) \\ National Institutes of Health (NIH) \\ Food and Drug Administration (FDA) \\ Acquired Immunodeficiency Syndrome (AIDS) \\ Non-Alcoholic Steato-Hepatitis (NASH) \\ Nonsteroidal Anti-Inflammatory Drug (NSAID) \\ Selective Serotonin Reuptake Inhibitors (SSRIs)
}

\section{Author contributions}

The author have accepted responsibility for the entire content of this submitted manuscript and approved submission.

\section{Research funding}

No funding involved.

\section{Employment or leadership}

Not applicable.

\section{Honorarium}

Not applicable.

\section{Competing interests}

NIL.

\section{Ethical approval}

The conducted review study is not related to either human or animal use. The present review study was accepted \& approved by the Sumandeep Vidyapeeth Institutional Ethics Committee (SVIEC), Sumandeep Vidyapeeth Deemed to be University, iparia.

\section{References}

1. Fernando Bessone. Non-steroidal anti-inflammatory drugs: What is the actual risk of liver damage? World J Gastroenterol 2010; 16: 5651-5661.

2. Fisher. Drug-Induced Liver Injury. Arch Pathol Lab Med 2015;139:876-7.

3. Dass Ervilla \& Patel. A prospective study of liver cirrhosis: An overview, prevalence, clinical manifestation $\&$ investigations 
in patients admitted to the medicine ward in a rural teaching hospital. Int J Sci Res 2018;7:614-6.

4. http://www.ema.europa.eu. Non-Clinical Guideline On DrugInduced Hepatotoxicity, Page 1-16. European Medicines Agency, London, 24 January 2008. Assessed online on 17-012017.

5. Aithal GP, Day CP. Nonsteroidal anti-inflammatory druginduced hepatotoxicity. Clin Liver Dis 2007;11:563-75, vi-vii.

6. William M. Lee, M.D. Drug-Induced Hepatotoxicity. $N$ Engl J Med 2003;349:474-85.

7. Singh A, Bhat TK, Sharma OP. Clinical Biochemistry of Hepatotoxicity. J Clinic Toxicol 2011;S4:001. doi:10.4172/2161-0495.S4-001.

8. Larrey D. Hepatotoxicity of herbal remedies. J Hepatol 1997;26:47-51.

9. Aida Ortega-Alonso, Camilla Stephens, M. Isabel Lucena, and Raúl J. Andrade. Case Characterization, Clinical Features and Risk Factors in Drug-Induced Liver Injury. Int $\mathrm{J} \mathrm{Mol} \mathrm{Sci}$ 2016:714; 1-22.

10. Sarah J. Mitchell and Sarah N. Hilmer. Drug-induced liver injury in older adults. Ther Adv Drug Saf 2010; 1: 65-77.

11. Ervilla E. Dass, Shah K. K. Paracetamol and conventional antimalarial drugs induced hepatotoxicity and its protection by methionine in rats. Indian Journal of Experimental Biology 2000; 38: 1138-1142.

12. Chitturi S, Farrell GC. Herbal hepatotoxicity: An expanding but poorly defined problem. J Gastroenterol Hepatol 2000:15:1093-9.

13. www.fda.gov. Food \& Drug Administration. CDER-PhRMAAASLD Conference 200: clinical white paper on drug-induced hepatotoxicity, November 2000: FDA Working Group Assessed online on 17-01-2017.

14. Khouryx T, Rmeilehx AA, Yosha L, Ariel A. Benson, Saleh Daher and Meir Mizrahix. Drug Induced Liver Injury: Review with a Focus on Genetic Factors, Tissue Diagnosis, and Treatment Options. J Clin Transl Hepatol 2015;3:99-108.

15. Zimmerman, H. J. Drug-induced liver disease: An overview. Seminars Liver Dis 1981;1: 93-103.

16. Chitturi S, George. Hepatotoxicity of commonly used drugs: nonsteroidal anti-inflammatory drugs, antihypertensives, antidiabetic agents, anticonvulsants, lipid-lowering agents, psychotropic drugs. J Semin Liver Dis 2002;22:169-83.

17. Routledge PA, O'Mahony MS, Woodhouse KW. Adverse drug reactions in elderly patients. Br J Clin Pharmacol 2004;57:121-6.

18. Harshad Devarbhavi. An Update on Drug-induced Liver Injury. J Clin Exp Hepatol 2012;2:247-59.

19. Singh D, Cho WC and Upadhyay G. Drug-Induced Liver Toxicity and Prevention by Herbal Antioxidants: An Overview. Front Physiol 2016;6:363. doi: 10.3389/fphys.2015.00363.

20. Mazza G, De Coppi P, Gissen P, Pinzani M. Hepatic regenerative medicine. J Hepatol 2015; 63:523-4.

21. Selden C, Spearman CW, Kahn D, Miller M, Figaji A, Erro E, et al. Evaluation of encapsulated liver cell spheroids in a fluidised-bed bioartificial liver for treatment of ischaemic acute liver failure in pigs in a translational setting. PLoS One 2013; 8: e82312.
22. Van de Kerkhove MP, Hoekstra R, Chamuleau RA, van Gulik TM. Clinical application of bioartificial liver support systems. Ann Surg 2004;240:216-30.

23. Allen JW, Hassanein T, Bhatia SN. Advances in bioartificial liver devices. Hepatol 2001;34:447-55.

24. Yu Y, Fisher JE, Lillegard JB, Rodysill B, Amiot B, Nyberg SL. Cell therapies for liver diseases. Liver Transpl 2012;18:921.

25. Fox IJ, Chowdhury JR, Kaufman SS, Goertzen TC, Chowdhury NR, Warkentin PI, et al. Treatment of the CriglerNajjar syndrome type I with hepatocyte transplantation. $N$ Engl J Med 1998; 338:1422-6.

26. Sokal EM, Smets F, Bourgois A, Van Maldergem L, Buts JP, Reding R, et al. Hepatocyte transplantation in a 4-year-old girl with peroxisomal biogenesis disease: technique, safety, and metabolic follow-up. Transplant 2003;76:735-8.

27. Katsutoshi Naruse, Wei Tang and Masatoshi Makuuchi. Artificial and bioartificial liver support: A review of perfusion treatment for hepatic failure patients. World J Gastroenterol 2007; 13:1516-21.

28. Ho MJ: Perspectives on tuberculosis among traditional Chinese medical practitioners in New York City's Chinatown. Culture. Med Psychiatr 2006;30:105-22.

29. Stickel F, Schuppan D: Herbal medicine in the treatment of liver diseases. Dig Liver Dis 2007;39:293-304.

30. World Health Organization Division of Traditional Medicine: General Guidelines for Methodologies on Research and Evaluation of Traditional Medicines Geneva; World Health Organization; 2000.

31. Prete AD, Scalera A, Iadevaia MD, Herbal Products: Benefits, Limits, and Applications in Chronic Liver Disease. EvidenceBased Complementary and Alternative Medicine 2012 (2012): 19 pages. https://doi.org/10.1155/2012/837939.

32. Biernat L, Hartleb M, Kochel A. Drug-induced liver damage a three-year study of patients from one gastroenterological department. Med Sci Monit 2002; 8: CR292-296.

33. Pandit A, Sachdeva T and Bafna P. Drug-Induced Hepatotoxicity: A Review. J Appl Pharm Sci 2012;02:233-43.

34. Chughlay MF, Kramer N, Werfalli M, Wendy Spearman, Mark Emmanuel Engel, and Karen Cohen. N-acetylcysteine for nonparacetamol drug-induced liver injury: a systematic review protocol. Syst Rev 2015;4:84.

35. Dass, Ervilla, \& Sattigeri, B. Hepatoprotective effect of DLmethionine on diclofenac-induced hepatotoxicity in albino rats: an experimental study. Int J Res Med Sci 2018; 6:802-7.

36. Ervilla D, Sattigeri B. Diclofenac-Induced Liver Toxicity in Albino Rats; Dose Dependent Study. Paripex-Indian J Res 2018;7.

37. Schapira D, Bassan L, Nahir A.M and Scharf Y. Diclofenacinduced hepatotoxicity. Postgraduate Med J 1986;62:63-5.

How to cite this article: Dass E. A brief review on Druginduced hepatotoxicity: Use of hepatoprotective agents. IP Int J Comprehensive Adv Pharmacol 2020;5(1):14-8. 